\title{
RF Radiometery Sensor Sensitivity and Detection Profile
}

\author{
AbdEl-Monem M. El-Sharkawy ${ }^{1,2}$ \\ Paul P. Sotiriadis ${ }^{1}$ \\ Department of Electrical Engineering ${ }^{1}$ \\ Department of Radiology ${ }^{2}$ \\ Johns Hopkins University \\ Baltimore, Maryland USA
}

\author{
Paul A. Bottomley ${ }^{1,2}$ \\ Ergin Atalar ${ }^{2,3}$ \\ Department of Electrical Engineering \\ Bilkent University, Turkey ${ }^{3}$
}

\begin{abstract}
Temperature sensing using microwave radiometry has proven value for non-invasively measuring the absolute temperature of tissues inside the human body. However, current clinical radiometers operate in $\mathrm{GHz}$ or infrared frequency ranges; this limits their depth of penetration since the human body is not "transparent" at these frequencies. To address this problem, we have previously designed and built an advanced, near-field radiometer operating at VHF frequencies $(64 \mathrm{MHz})$ with a $\sim 100 \mathrm{KHz}$ bandwidth. The radiometer has performed accurate temperature measurements to within $\pm 0.1^{\circ} \mathrm{C}$, over a tested physiological range of $28-40^{\circ} \mathrm{C}$ in saline phantoms whose electric properties match those of human tissue. In this work we analyze radiofrequency (RF) coil designs suitable for RF Radiometry. We investigate the coil profile sensitivity to look where temperature information is coming from and the depth of penetration associated with the receiver used. We also look into the virtues of using multi-turn coils versus single loop coils. We conclude that by using multi-turn coils the received noise signal is more sensitive to sample noise and temperature can be estimated more accurately especially with the use of smaller receivers.
\end{abstract}

\section{INTRODUCTION}

Over the past 30 years, radiometry has been used to measure the temperature of biological tissues in human and animal models. Being a passive detection modality, it does not require exposure to radiation and so it is both safe and entirely nonhazardous. Because fast-growing tumors can exhibit local temperature elevations over surrounding normal tissues, microwave radiometry

This work was supported by the National Institute of Health (NIH) grants R01HL57483, and R01RR15396.

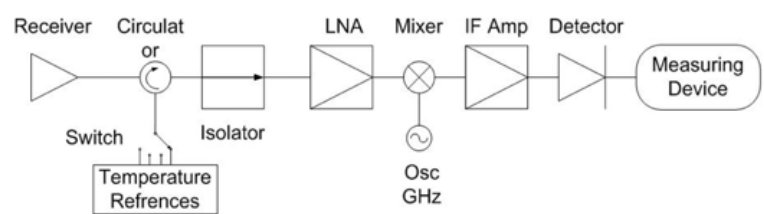

Figure 1. Typical microwave radiometer architecture: the signal (noise) received by the antenna passes through the circulator and the isolator that compensate for any mismatch. The signal is then amplified by a low noise amplifier and then downconverted to a lower frequency and filtered. The measuring device at the end estimates the RMS power of the signal (noise).

has shown promise as a possible diagnostic tool for early breast cancer detection [1].

Of importance to the diagnostic utility of radiometry is the depth of tissue whose temperature measurements are being sought. The depth of penetration of EM radiation in human tissue decreases with frequency. Thus, radio frequencies (RF) below $200 \mathrm{MHz}$ are best suited for radiometry of tissues lying deep in the body. Current clinical microwave radiometers operate at the $\mathrm{GHz}$ frequency range. Their architecture is typically based on Dicke's 1946 radiometer [2]. They are commonly built with a receiving antenna connected to a circulator and/or an isolator to reduce inaccuracy due to load variation (Figure 1).

In this work we analyze the radiometer antenna sensitivity for a radiometer we have previously built 
[3] that operates at $64 \mathrm{MHz}$ in order to achieve adequate depth of penetration and be compatible with $1.5 \mathrm{~T}$ MRI scanners [3]. Accurately measuring radiated noise power at low RF frequencies (and small bandwidths) is challenging because the received signal has relatively less available power and the antenna operates in the near-field making the reception very sensitive to movement and environmental changes. Also, isolators and circulators are impractical due to their physical sizes at such frequencies.

In our system, the antenna (coil) used for receiving noise radiation is precisely matched with an accuracy of $0.05 \Omega$ to $50 \Omega$ to compensate for load variations, using automatic vector impedance sensing. After impedance-matching the antenna is switched to an amplifier where the received noise power is amplified and measured. Based on the received signal the temperature of a body lying in the antenna's (near-field) region of sensitivity is estimated. As in standard microwave radiometers, our system requires calibration against known temperature loads. In order to keep system calibrated parameters as stable as possible we investigate the use of multi-turn coils versus single loop coils. We demonstrate the measurement of absolute temperatures on a phantom of comparable EM properties to biological tissue over a physiological temperature range.

\section{THEORY}

The main concept of RF radiometry is based on the well-known theory of black body radiation as described by Nyquist [4]. The noise voltage variance generated in a loop RF coil can be expressed as

$$
\begin{aligned}
& <V_{m}^{2}>=4 k T_{\text {sample }} B R_{\text {sample }}+4 k T_{\text {comp }} B R_{\text {comp }} \\
& \ldots \ldots \ldots . \\
& \ldots \ldots+V_{n}^{2}>+<V_{c}^{2}>
\end{aligned}
$$

Where $\left\langle\mathrm{V}_{\mathrm{m}}{ }^{2}\right\rangle$ is the variance of the measured thermal noise open circuit voltage; $\left\langle\mathrm{V}_{\mathrm{n}}^{2}\right\rangle$ and $\left\langle\mathrm{V}_{\mathrm{c}}{ }^{2}\right\rangle$ are the noise contributions from sample loading and coil components respectively; $\mathrm{R}_{\text {sample }}$ and $\mathrm{R}_{\text {comp }}$ sample and coil components real resistances; $\mathrm{k}$ is Boltzman's constant; and $\mathrm{B}$ is the bandwidth of the receiving system.
After the coil is tuned and matched and amplified through the receiver chain the measured noise power is

$$
P_{m}=\left(1-\Gamma^{2}\right) G(\Gamma) k T B+N(\Gamma)+P_{c}
$$

where $\Gamma$ is the reflection coefficient at the antenna-amplifier interface; $G$ is the available system gain; $\mathrm{N}$ is the noise power added by the system and $\mathrm{P}_{\mathrm{m}}$ is the measured noise power and $\mathrm{P}_{\mathrm{c}}$ is noise power contributed by coil components.

In lumped form this relation can be written as :

$$
P=\alpha(\Gamma) T+\beta(\Gamma)(3)
$$

Where $\alpha$ and $\beta$ are unknown lumped system parameters. When $\Gamma$ is constant, the system parameters $(\alpha, \beta)$ are fixed and can be derived by calibration using known temperature loads. In order to reduce the variation in $\beta$ (and thus in temperature estimation variance) the sensor would be designed to minimize $P_{c}$ with respect to the total measured noise power. $R_{c}$ can be calculated as wire resistance and resistance added by capacitors to tune the coil. This would be achieved by minimizing the ratio of $R_{\text {comp }} / R_{T}$; where $R_{T}=R_{\text {sample }}+R_{\text {comp }}$.

\section{METHODS}

Electromagnetic simulations using the Method of Moments (FEKO software) is used to simulate a homogenous sample loading for a $6 \times 6 \mathrm{~cm}$ square coil tuned at $64 \mathrm{MHz}$. The total sample resistance is calculated using

$$
R_{\text {sample }}=\int_{V} \sigma(r, \omega)|E(r, \omega)|^{2} d r
$$

Where $\sigma$ is the body's conductivity, (spatial and temperature dependent); and $\mathrm{E}$ is the (total) electric field distribution of the antenna for unit current excitation.

The coil electrical components resistance is calculated as:

$$
R_{\text {comp }}=R_{\text {cap }}+\rho \frac{L}{2 \delta_{s} W}
$$



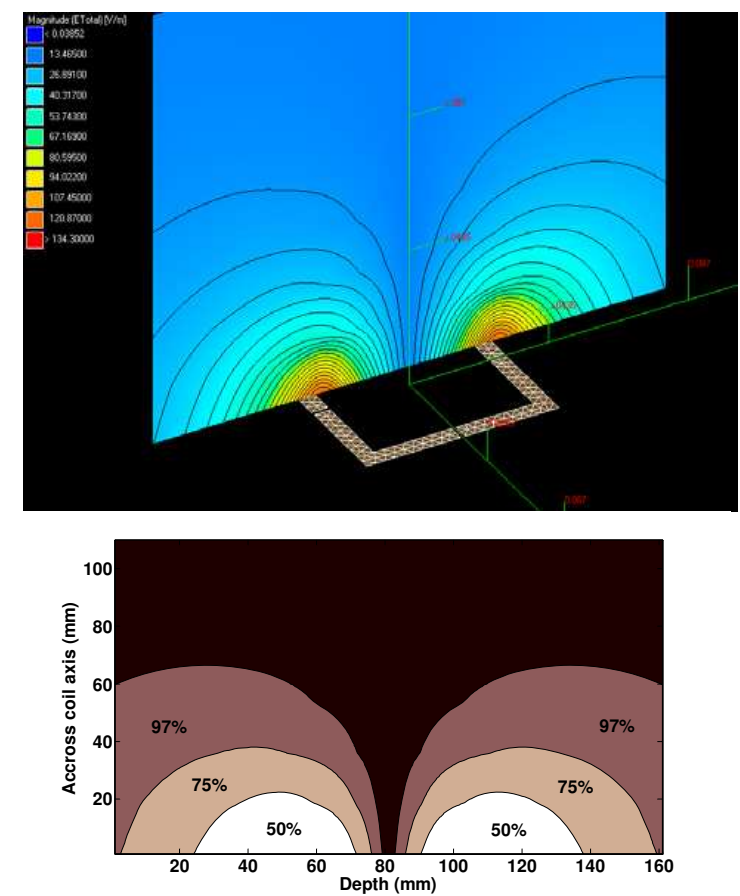

Figure 2. Top; electric field distribution in phantom for the $6 \times 6 \mathrm{~cm}$ loop square coil. Bottom; Coil sensitivity profile for the same coil. Depth of penetration is calculated to be $\sim 6 \mathrm{~cm}$.

Where $\rho$ is copper recesistivity $\left(1.7 \times 10^{-8} \Omega \mathrm{m}\right), \mathrm{L}$ is the length of the loop, $\mathrm{W}$ is the width of the copper tracks $(5 \mathrm{~mm})$ and $\delta_{\mathrm{s}}[5]$ is the skin depth at $64 \mathrm{MHz}$ (f) for copper obtained by;

$$
\delta_{s}=\sqrt{\frac{1}{\pi f \mu \sigma}}(6)
$$

Where $\mu$ is permeability of free space and $\sigma$ is copper conductivity. $\delta_{\mathrm{s}}$ is calculated to be $8.2 \mu \mathrm{m}$.

The temperature measured by an RF radiometer is

$$
T_{\text {meas }}=\frac{\int_{r} T(r) P_{d}(r) d r}{\int_{r} P_{d}(r) d r}, P_{d}(r)=\sigma(r)|E(r)|^{2}
$$

Where $\mathrm{P}_{d}(\mathrm{r})$ : antenna receiving (power) pattern calculated using unit current excitation. By reciprocity law, the antenna receiving pattern in the body is identical to that of the power absorbed, the specific absorption rate (SAR), when using the radiometer's antenna as a transmitter. By looking at the receiving power pattern and calculating integration ratio of partial volume over total space ratio (Profile) we are able to estimate sensitivity profile and depth of penetration of the radiometric sensor. Where;
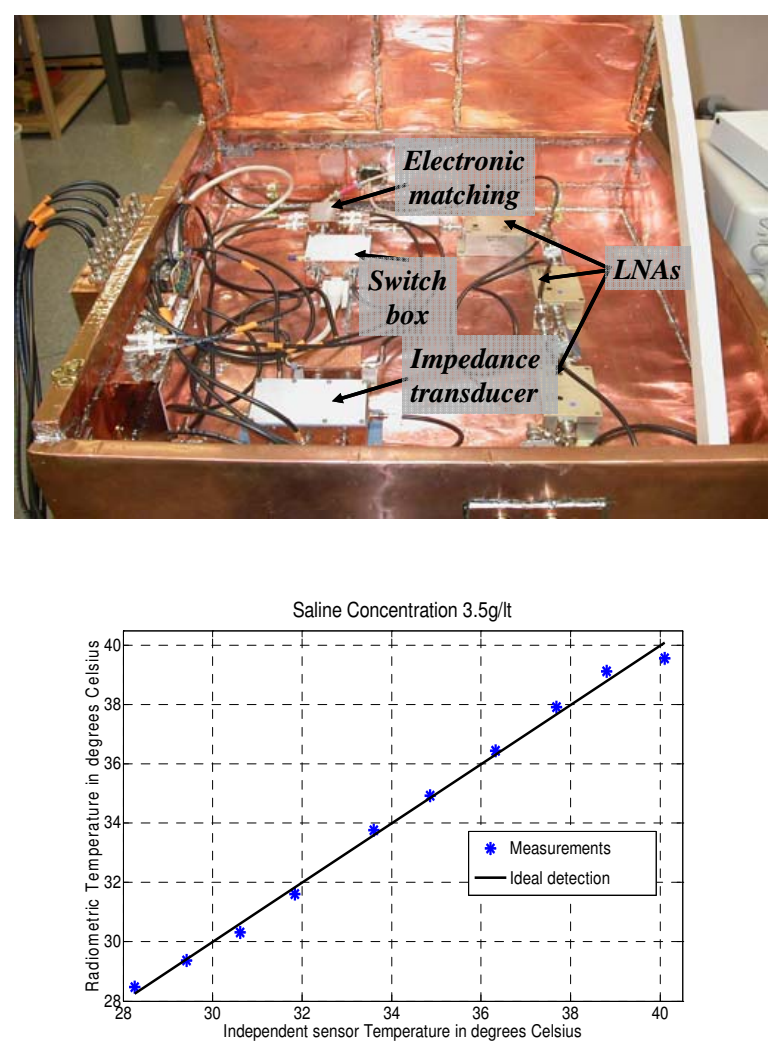

Figure 3. Top; Picture of part of our RF radiometer. Bottom; Temperature measured from noise power vs. temperature measured with accurate fiber optic thermometer.

$$
\operatorname{Pr} \text { ofile }(\%)=\frac{\int_{V} P_{d}(r) d r}{\int_{r} P_{d}(r) d r} \times 100
$$

\section{RESUlts}

Simulation results for a single and a double turn coil are tabulated in table 1 . The single and 


\begin{tabular}{|l|l|l|l|}
\hline & $\mathrm{R}_{\text {sample }}$ & $\mathrm{R}_{\text {coil }}$ & Rcoil/Rtotal \\
\hline Single loop & 1.25 & 0.15 & $\sim 8.5 \%$ \\
\hline Double loop & 5 & 0.2 & $3.8 \%$ \\
\hline
\end{tabular}

Table $1 . \mathrm{R}_{\text {sample }}$ values resulting from EM Method of Moments simulation using homogenous saline loading of conductivity $0.7 \mathrm{~S} / \mathrm{m}$ for a $6 \times 6 \mathrm{~cm}$ square coil. The theoretical values of $\mathrm{R}_{\text {coil }}$ are also tabulated.

double loop coils were tuned using four series capacitors. Capacitance resistance was estimated from data sheet reported quality factor $(\mathrm{Q})$ values as $0.025 \Omega$. Results suggest that the sample resistance quadruples as the number of coil turns doubles. Calculations are tabulated in Table 1. The total coil component contribution decreases by a factor of 2. This suggests that using a multi-turn RF coil is more suitable for radiometric measurements.

Shown in figure 2 are the results for the profile sensitivity of the $6 \times 6 \mathrm{~cm}$ radiometric sensor. It is shown that the depth of penetration for $100 \%$ detection is $\sim 6 \mathrm{~cm}$.

A single loop coil was tested on the radiometric system with heated water phantoms prepared with different concentrations of saline. Phantom temperature was accurately controlled and monitored via an independent fiber-optic temperature sensing device connected to a heater. The radiometer was first calibrated against references of known temperature. After calibration, radiometric temperature measurements were performed and compared with the independent sensor measurements (Figure 3). The calculated RMS error over four studies shows an accuracy of $\pm 0.1^{0} \mathrm{C}$ in temperature estimation [3].

\section{CONCLUSION}

We have demonstrated through phantom studies that with the use of single loop antenna, using continuous RF impedance sensing, matching and calibration, RF radiometry can be used to detect absolute temperature with an accuracy of $\pm 0.1^{\circ} \mathrm{C}$ over a physiological range [3]. The loop antenna is very sensitive near the winding (Figure 2) and has a temperature detection sensitivity profile corresponding to their power receiving profile. As the sample coil resistance is proportional to the coil size, using multi-loop coils are more advantageous over single loop ones especially when using smaller coils. The design of different antenna topologies for better profile sensitivity and detection accuracy is under investigation. Possible future design includes the use of coil arrays and noise correlation profile [6] to obtain spatially resolved radiometric measurements.

\section{ACKNOWLEDGMENT}

The authors would like to thank Dr. Marion Lee Edwards, Director of RF Eng. group APL, for insightful technical comments. Special thanks go to Dr. William Edelstein and Ananda Kumar, Johns Hopkins University, for ideas on how to calculate coil resistance.

\section{REFERENCES}

[1] K. Carr, "Microwave Radiometry: Its Importance to The Detection of Cancer", IEEE Trans. on Microwave Theory and Techniques, Vol. 37, No. 12, P.1862-1869, Dec. 1989.

[2] R. Dickie, "The Measurement of Thermal Radiation at Microwave Frequencies", Rev. Sci. Instr., Vol. 17, P. 268-275, July 1946.

[3] A. El-Sharkawy, P. Sotiriadis, P. Bottomley, E. Atalar, "Absolute Temperature Monitoring using $R F$ Radiometry in the MRI Scanner", IEEE Trans. on Circuits \& Systems-I, Vol. 53, No. 11, Nov. 2006, pp. 2396-2404.

[4] H. Nyquist, "Thermal Agitation of Electric Charge in Conductors", Physical Review, Vol. 32, P. 110-112, July 1928.

[5] D. Pozar, "Microwave Engineering”, John Wiley \& Sons Inc., 1998, pp. 19-21.

[6] T. M. Taves, and S. B. King, "In Vivo Conductivity Measurement using MRI based Noise Tomography at 3T", proceedings of ISMRM 2007. 